\title{
Assessing nodules detected in lung cancer screening: the value of positron emission tomography
}

\author{
Jann Mortensen
}

Affiliation: Dept of Clinical Physiology, Nuclear Medicine and PET, Rigshospitalet, Copenhagen University Hospital, Copenhagen, Denmark.

Correspondence: Jann Mortensen, Dept of Clinical Physiology, Nuclear Medicine and PET, Rigshospitalet, Blegdamsvej 9, DK-2100 Copenhagen Ø. Denmark. E-mail: jann.mortensenवregionh.dk

-

@ERSpublications

PET has the highest accuracy in differential diagnosis of upper lobe, solid $>10 \mathrm{~mm}$ or partlysolid $>15 \mathrm{~mm}$ nodules http://ow.ly/DKaur

Lung cancer is the leading cause of death worldwide. Screening for lung cancer may improve the poor prognosis by detecting the cancer at an early stage where radical surgery still is possible. Several randomised clinical trials using low-dose computed tomography (CT) have been launched in high-risk smokers or former smokers in the USA and Europe [1-3]. The National Lung Screening Trial (NLST) randomised 53454 subjects to undergo annual CT scans or chest radiographies for 3 years, and demonstrated that overall and lung cancer specific mortality was reduced in the CT arm [1]. For one case of death from lung cancer to be prevented, the number of patients that needed to be screened using CT compared with chest radiography was 320 [3]. The final mortality data testing of $>32000$ individuals participating in the seven European randomised lung cancer CT screening trials (EUCT), where the control groups had no chest radiography, is expected in 2015 [2].

The drawbacks of CT screening programmes also need to be taken into account. Most subjects eligible for screening will never develop lung cancer, but will be exposed to different potential harms such as distress while waiting for the test result, and the distress and complications related to the many false positive tests, which will require subsequent invasive diagnostic procedures in some individuals. In addition, the radiation exposure from low-dose CT in all participants and from additional imaging tests in many participants is a concern $[4,5]$.

In the baseline round (prevalence), a positive nodule was detected in $8-30 \%$ of the participants in the CT groups of the randomised screening trials [3]. The false positive prevalence was $86-95 \%$ and lung cancer was detected in $0.8-2.2 \%$ of the participants. The variation in the results is partly due to differences in the screened populations, the criteria for defining a positive nodule and the follow-up procedures.

Different management strategies are applied to discriminate between malignant and benign nodules, so the large number of false positives and number of subsequent workup procedures can be reduced. The second-line procedures most often comprise repeat CT scans after 3 months or at a later time-point to evaluate possible growth and/or change in morphology conspicuous of malignancy $[4,6]$.

Functional imaging by ${ }^{18} \mathrm{~F}$-fluorodeoxyglucose (FDG) positron emission tomography (PET) can also be used to evaluate if there is increased FDG uptake in a nodule indicating malignancy $[6,7]$ as well for staging lung cancer [8]. PET is a quicker method to diagnose an indeterminate nodule as malignant or benign compared with waiting some months for a follow-up CT [7]. Recently, predictive tools based on patient and nodule characteristics have been introduced to estimate the cancer risk in screening studies [9].

Received: Oct 172014 | Accepted: Oct 232014

Conflict of interest: None declared

Copyright OERS 2015 
These approaches can be used separately or together $[6,7]$. A lung cancer is highly suspected if the nodule is growing with a volume-doubling time (VDT) $<365$ days (or $<400$ days) and/or if it is PET positive $[4,6]$. A nodule is likely to be benign or represent an indolent cancer if it is not growing (or growing very slowly) or if it is PET negative, and usually a new CT scan is recommended after 1 year. However, neither the VDT nor PET methods can be absolutely relied upon, because both can give false negatives and positives. Some benign lesions may demonstrate growth. Likewise, PET can be positive in inflammatory/infectious lesions (e.g. granulomas), while some cancers can be slow growing and be PET negative $[10,11]$.

This issue of the European Respiratory Journal includes an article addressing the value of PET in the diagnostic workup of screening-detected lung nodules. The article by Veronesi et al. [12] is based upon 383 nodules that were examined by PET/CT over the first 6 years of the Italian nonrandomised single-centre Continuous Observation of Smoking Subjects (COSMOS) early detection trial for lung cancer. These nodules were read as indeterminate on low-dose CT. To qualify for PET/CT an indeterminate nodule should be noncalcified solid or partly solid $>8 \mathrm{~mm}$ without appearing clearly benign, and a follow-up CT scan was performed 1 year later. Nodules between 5 and $8 \mathrm{~mm}$ were initially controlled with CT after 3 or 6 months and if growing a PET/CT was performed (some nodules in this group could be $<8 \mathrm{~mm}$ ). Growing or PET-positive nodules suspicious for malignancy underwent minimally invasive surgical biopsy or fine-needle aspiration and additional interventions. The PET findings were retrospectively compared with pathological findings (either cancer or benign) or follow-up CT (benign if no growth for $\geq 2$ years). By virtue of the large number of included nodules with different sizes, densities, pathological types, location in the lungs, and detected at the prevalence or incidence rounds, this study sheds light on some of the strengths and limitations of PET to differentiate between malignant and benign indeterminate lung nodules $[6,7,11]$.

\section{Is the accuracy of PET dependent on nodule size and type?}

The accuracy of PET is heavily dependent on the size of the nodule if it is approaching the resolution of the scanner. The newest PET scanners have a spatial resolution of 4-5 $\mathrm{mm}$ and the older versions have a spatial resolution $\sim 8-10 \mathrm{~mm}$. Since solid tumours generally have a higher cell density than both subsolid and nonsolid tumours, the FDG uptake and reliability of PET is higher in solid than in nonsolid tumours of the same size [11]. VERONESI et al. [12] found that the accuracy (and sensitivity) for solid nodules was $76 \%$ (51\%), 92\% (86\%) and $88 \%$ (98\%) for nodules $<10 \mathrm{~mm}, 10-15 \mathrm{~mm}$ and $\geq 15 \mathrm{~mm}$, respectively. The figures were much lower for the partly or nonsolid nodules combined: $53 \%$ (17\%), 47\% (25\%) and $70 \%$ (64\%) for nodules $<10 \mathrm{~mm}, 10-15 \mathrm{~mm}$ and $\geq 15 \mathrm{~mm}$, respectively. Hence, the performance of PET is better in solid nodules $\geq 10 \mathrm{~mm}$ and partly-solid nodules $>15 \mathrm{~mm}$, while PET cannot be relied upon in nonsolid nodules no matter the size $[11,12]$.

The American College of Chest physicians suggests PET should be performed to characterise indeterminate solid nodules $>8 \mathrm{~mm}$ if the pretest probability of malignancy is low to moderate (5-65\%) [13]. CT surveillance is recommended instead if the pretest probability is very low $(<5 \%)$ or in the case of a low-to-moderate pretest probability with a negative PET scan.

\section{Is the accuracy of PET the same for all tumour types?}

Most lung cancer types have an increased FDG uptake, but the degree varies from no uptake to a very high uptake $[11,12]$. Sensitivity was only $53 \%$ for adenocarcinomas, whereas it was $82-92 \%$ for the other cancer types [12]. Squamous cell carcinomas, nonsmall cell lung cancer and small cell lung cancer had the highest uptake values (maximum standardised uptake value), carcinoids had an intermediate uptake and adenocarcinomas had the lowest uptake, although the ranges overlapped, so it is not possible to use FDG uptake as a surrogate for histological typing.

\section{Is the accuracy of PET dependent on the localisation of a nodule?}

Since patients cannot hold their breath during the 1-5 min long acquisition times of PET per bed position, the nodules move during acquisition, especially in the basal parts of the lung. These blurring phenomena will reduce the signal of the FDG uptake into a nodule. This might be partly avoided if PET acquisition is gated to the respiration at the expense of substantially increasing the acquisition time. VERONESI et al. [12] found lower sensitivity in the lower lobes (53\%) compared with the upper lobes (71\%), but no difference in the performance of PET for nodules located in the right and left lung.

\section{Can interpretation of PET be based on a visual reading only?}

A clinical PET scan is most often interpreted by a visual analysis of the tracer uptake compared with the physiological background or by a semi-quantitative analysis (standardised uptake value), which is especially needed when monitoring the effects of treatment. The methods can also be used together, and both have their pros and cons. Veronesi et al. [12] analysed all nodules by visual interpretation and by 
three maximum standardised uptake value thresholds (1.5, 2.0 and 2.5) for calling a nodule malignant. While sensitivity and specificity varied inversely, with decreasing sensitivity and increasing specificity at higher thresholds, the accuracy remained the same, but was lower compared with the visual analysis. Whether a correction for partial volume could have improved performance of the standardised uptake value analysis was not reported. The finding of this study is in keeping with other studies of lung nodules, in which a visual PET analysis was reliable in small nodules $[6,7,14]$.

\section{Is the accuracy of PET similar at baseline and later rounds of screening?}

More nodules are found in the first baseline round compared with later screening rounds, where it is possible to correlate to the previous scans, and the relative risk of cancer in a suspicious nodule is higher in later rounds [3]. PET had a higher diagnostic accuracy for nodules detected at the baseline screening (88\%) than at later rounds (between $50 \%$ and $80 \%$ in each round), in line with other studies on lung cancer screening $[6,12]$. This is probably because cancers found in later rounds are smaller and more often nonsolid, where the performance of PET is less favourable $[11,12]$.

\section{In which settings is PET most reliable?}

PET has the highest accuracy for differential diagnosis of indeterminate nodules detected at baseline, that are solid $>10 \mathrm{~mm}$ or partly-solid $>15 \mathrm{~mm}$, and localised in the upper lobes. Where PET is less reliable other tests, such as VDT measurements, are probably better [12]. Ashraf et al. [6] showed, in a study on 54 nodules from the Danish Lung Cancer Screening Trial, that both the PET uptake and the VDT were independently associated with malignancy, and the tests could be combined. The diagnostic value was stronger if the methods were concordant. An indeterminate nodule was very likely to be malignant if VDT was short ( $<365$ days) and PET was positive, whereas the nodule was probably benign if VDT was long and PET was negative [7]. The data suggested an intermediate-to-high probability of cancer if the tests were discordant [6]. Larger confirmatory studies of this concept are needed. It also remains to be determined to what extent the diagnostic value of PET found in lung cancer trials can be applied to patients in clinical practice with incidentally detected small lung nodules with an intermediate probability for lung cancer.

\section{References}

1 Aberle DR, Adams AM, Berg CD, et al. Reduced lung-cancer mortality with low-dose computed tomographic screening. N Engl J Med 2011; 365: 395-409.

2 Field JK, van Klaveren R, Pedersen JH, et al. European randomized lung cancer screening trials: Post NLST. J Surg Oncol 2013; 108: 280-286.

3 Shlomi D, Ben-Avi R, Balmor GR, et al. Screening for lung cancer: time for large-scale screening by chest computed tomography. Eur Respir J 2014; 44: 217-238.

4 Horeweg $\mathrm{N}$, van der Aalst CM, Vliegenthart R, et al. Volumetric computed tomography screening for lung cancer: three rounds of the NELSON trial. Eur Respir J 2013; 42: 1659-1667.

5 Aggestrup LM, Hestbech MS, Siersma V, et al. Psychosocial consequences of allocation to lung cancer screening: a randomised controlled trial. BMJ Open 2012; 2: e000663.

6 Ashraf $\mathrm{H}$, Dirksen A, Loft A, et al. Combined use of positron emission tomography and volume doubling time in lung cancer screening with low-dose CT scanning. Thorax 2011; 66: 315-319.

7 Bastarrika G, García-Velloso MJ, Lozano MD, et al. Early lung cancer detection using spiral computed tomography and positron emission tomography. Am J Respir Crit Care Med 2005; 171: 1378-1383.

8 Fischer B, Lassen U, Mortensen J, et al. Preoperative staging of lung cancer with combined PET-CT. N Engl J Med 2009; 361: 32-39.

9 McWilliams A, Tammemagi MC, Mayo JR, et al. Probability of cancer in pulmonary nodules detected on first screening CT. N Engl J Med 2013; 369: 910-919.

10 Veronesi G, Maisonneuve P, Bellomi M, et al. Estimating overdiagnosis in low-dose computed tomography screening for lung cancer: a cohort study. Ann Intern Med 2012; 157: 776-784.

11 Nomori H, Watanabe K, Ohtsuka T, et al. Evaluation of F-18 fluorodeoxyglucose (FDG) PET scanning for pulmonary nodules less than $3 \mathrm{~cm}$ in diameter, with special reference to the CT images. Lung Cancer 2004; 45 : $19-27$.

12 Veronesi G, Travaini LL, Maisonneuve P, et al. Positron emission tomography in the diagnostic work-up of screening-detected lung nodules. Eur Respir J 2015; 45: 501-510.

13 Gould MK, Donington J, Lynch WR, et al. Evaluation of individuals with pulmonary nodules: when is it lung cancer? Diagnosis and management of lung cancer, 3rd ed: American College of Chest Physicians evidence-based clinical practice guidelines. Chest 2013; 143: Suppl. 5, e93S-e120S.

14 Nomori H, Watanabe K, Ohtsuka T, et al. Visual and semiquantitative analyses for F-18 fluorodeoxyglucose PET scanning in pulmonary nodules $1 \mathrm{~cm}$ to $3 \mathrm{~cm}$ in size. Ann Thorac Surg 2005; 79: 984-988. 